This item was submitted to Loughborough's Research Repository by the author.

Items in Figshare are protected by copyright, with all rights reserved, unless otherwise indicated.

\title{
Model to predict the mechanical behaviour of oriented rigid PVC
}

PLEASE CITE THE PUBLISHED VERSION

PUBLISHER

(C) Maney

LICENCE

CC BY-NC-ND 4.0

\section{REPOSITORY RECORD}

Hitt, David J., and Dmytro Miroshnychenko. 2006. "Model to Predict the Mechanical Behaviour of Oriented Rigid PVC". figshare. https://hdl.handle.net/2134/2234. 


\title{
L Loughborough University
}

This article was published in the journal, Plastics, Rubbers and Composites, 34 (2005)

\section{MODEL TO PREDICT THE MECHANICAL BEHAVIOUR OF ORIENTED RIGID PVC}

\author{
D.J. Hitt ${ }^{1}$ and Dmitri Miroshnychenko ${ }^{2}$ \\ ${ }^{1}$ IPTME, Loughborough University, Loughborough, Leicestershire LE11 3TU, UK \\ ${ }^{2}$ Department of Mathematics, University of Glasgow, 15 University Gardens, Glasgow G12 8QW, UK
}

\begin{abstract}
The mechanical properties of PVC sheets can be modified substantially by both uniaxial and biaxial stretching of the material above its glass transition temperature. Previous experimental studies have established a clear pattern in the relationship between tensile properties of oriented PVC products and imposed strains. Several mathematical models have been scrutinised to assess whether the established pattern of behaviour can be modelled and predicted. Of these, "the filament theory", proposed by Turner, emerged as the best candidate. The filament theory has been refined and developed further into "the composite model". In its present form it gives a good correlation between predicted and measured yield stress values of oriented rigid PVC sheets and is also capable of predicting the "established pattern" of property dependence upon imposed strain.
\end{abstract}

\section{INTRODUCTION}

The mechanical properties of rigid PVC can be improved substantially by stretching at temperatures above the glass transition temperature of the polymer, $T_{\mathrm{g}}$, which is typically in the range $75^{\circ} \mathrm{C}-80^{\circ} \mathrm{C}$. As $\mathrm{PVC}$ has its peak elongation at $90^{\circ} \mathrm{C}$ [1-4], the mechanical properties of the oriented product can be optimised by drawing at this temperature. After stretching, the material must be held under restraint to ensure that the oriented structure is "frozen-in" on cooling below $T_{\mathrm{g}}$ to ambient temperature. Removal of the restraint at the draw temperature will enable the stretched material to recover its original dimensions. Experimental data gathered from previous studies at IPTME [5-8] has shown the tensile strength properties of oriented PVC sheets are related to the strains imposed at $90^{\circ} \mathrm{C}$ in a very systematic way. This behaviour is clearly ideally suited to the application of mathematical modelling. In pursuit of this goal several mathematical models from the literature have been evaluated. Of these, one particular treatment, "the filament theory" proposed by Turner [9], emerged as having the best potential to achieve the goal. This theory has been refined and developed into the composite model by Miroshnychenko et al. $[10,11]$. To reflect the application of "the filament theory" to oriented PVC and its ongoing refinement this paper is divided into three main sections: First, a selection of data is presented that illustrates the systematic pattern of tensile strength versus imposed strain for oriented rigid PVC. Second, the filament theory is presented in terms of its concept, its key assumptions and its use to predict the properties of oriented PVC. The third section will examine the scope for further refinements to the filament theory and composite model and their validation. 


\section{THE DEPENDENCE OF MECHANICAL STRENGTH OF ORIENTED RIGID PVC UPON IMPOSED STRAINS}

From the studies at IPTME [5-8] on the effects of orientation on the properties of rigid PVC it has been found that both yield strength and tensile strength follow the same pattern when properties are plotted against planar strain. (This is essentially the product of the draw ratios applied to the material, for example, PVC sheets stretched uniaxially to draw ratios of $3.0 \times 1.0$ and equally biaxially to $1.73 \times 1.73$ have the same planar strain of 3.0.) Figures 1 and 2 illustrate the systematic pattern for both tensile properties. In both figures a selection of draw ratios have been included adjacent to data points: for unequal draw ratios the direction of property measurement is indicated by the underlined draw ratio. It can be seen that at a given planar strain tensile strength can be ranked according to the stretching mode: uniaxial (machine direction, MD) > unequal biaxial (major draw direction) $>$ equal biaxial $>$ unequal biaxial (minor draw direction) $>$ uniaxial (transverse direction, TD). Note: Brady [12] advocated the use of the planar strain parameter to correlate data of oriented products and Figure 1 is consistent with his findings on the yield stress of uniaxially and biaxially oriented PVC.

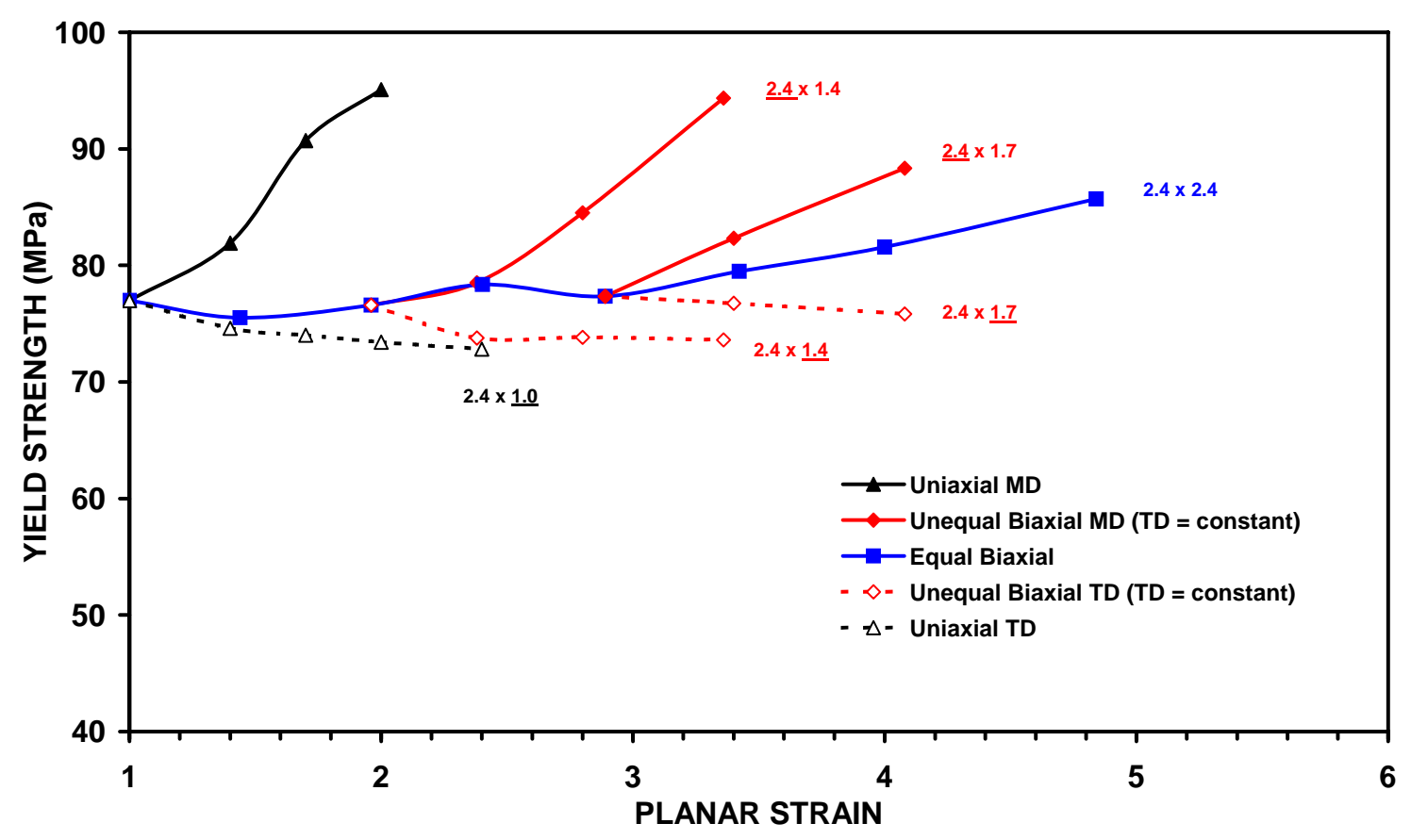

Figure 1: Dependence of yield strength of oriented PVC-U on strains imposed at $90^{\circ} \mathrm{C}$ 


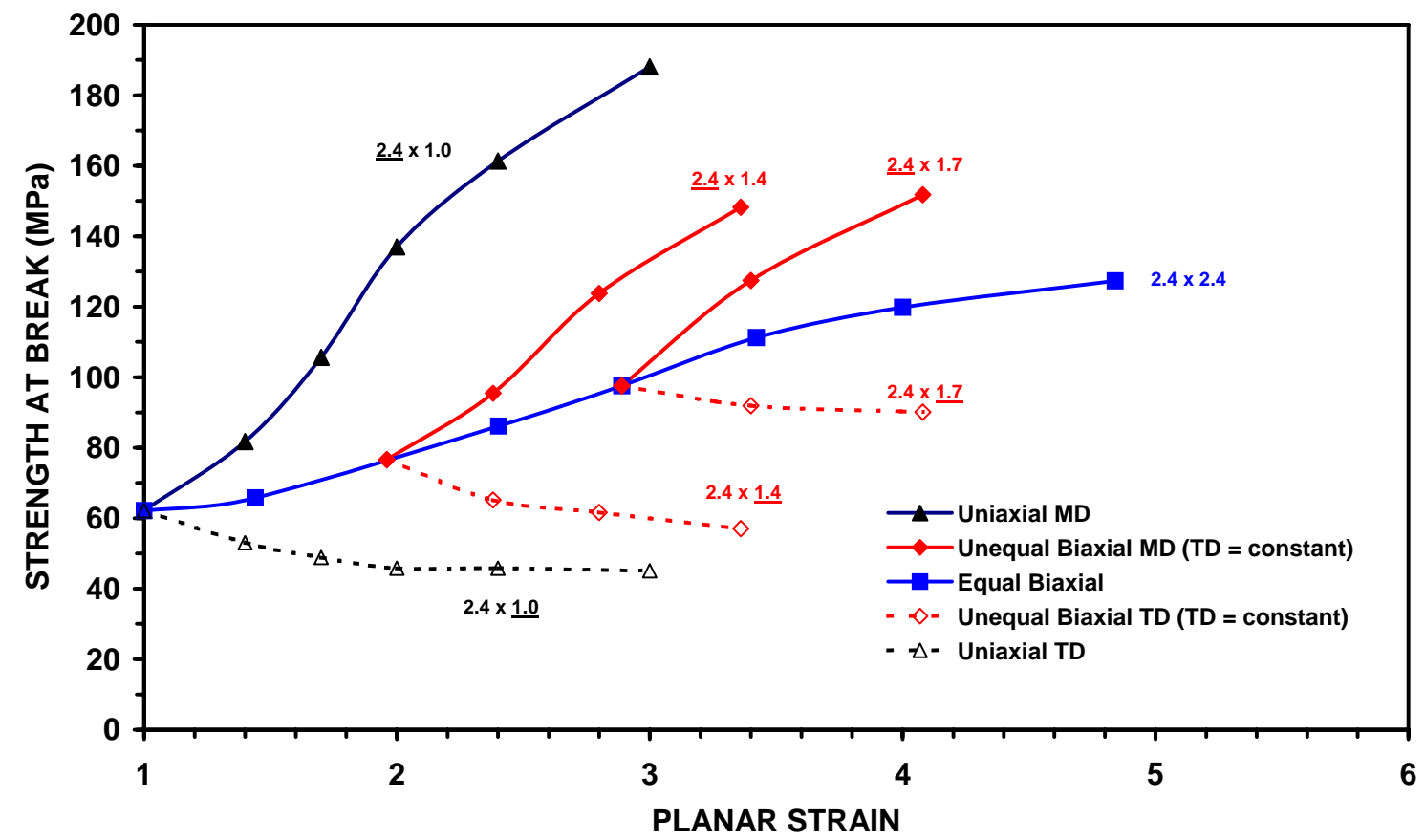

Figure 2: Dependence of strength at break of oriented PVC-U on strains imposed at $90^{\circ} \mathrm{C}$

It must be pointed out that the uniaxially oriented sheets were produced under a constant width stretching mode, i.e., in effect a low strain was applied in the TD so that the draw ratio in this direction was maintained at 1.0 .

The patterns of behaviour can be understood in terms of the physical structure of PVC that is well known. This effectively consists of a three-dimensional amorphous network of long chains linked together by very small crystallites, of around 10nm in size, that typically account for $10 \%$ of the structure. When the material is stretched above $T_{\mathrm{g}}$ the long chains are progressively straightened and become loosely aligned in the direction of the applied draw. At the same time the crystallites are drawn into the plane of the sheet. Naturally, the greatest chain alignment, and consequently the greatest enhancement in tensile strength, will occur in the MD of a material that has been highly oriented uniaxially. There is, of course a down side, little chain alignment is produced in the TD and this results in very poor tensile strength relative to the MD. In PVC products this differential in mechanical performance may be advantageous or a serious problem. 
Replication of the patterns of Figures 1 and 2 became the prime objective of work to develop a mathematical model. Allied to this the model needs to recognise the factors that modify the yield and stress-strain behaviour of rigid PVC. A tensile sample of unoriented rigid PVC at ambient temperature will yield by the formation of a neck. This is reflected by a sharp drop in the measured load-extension curve: the yield point. The necked region then grows until the whole gauge length of the sample has undergone necking. This is then followed by uniform deformation of the gauge length until failure occurs. However, as the test temperature approaches $T_{\mathrm{g}}$, yield behaviour and subsequent deformation is modified significantly (Figure 3).

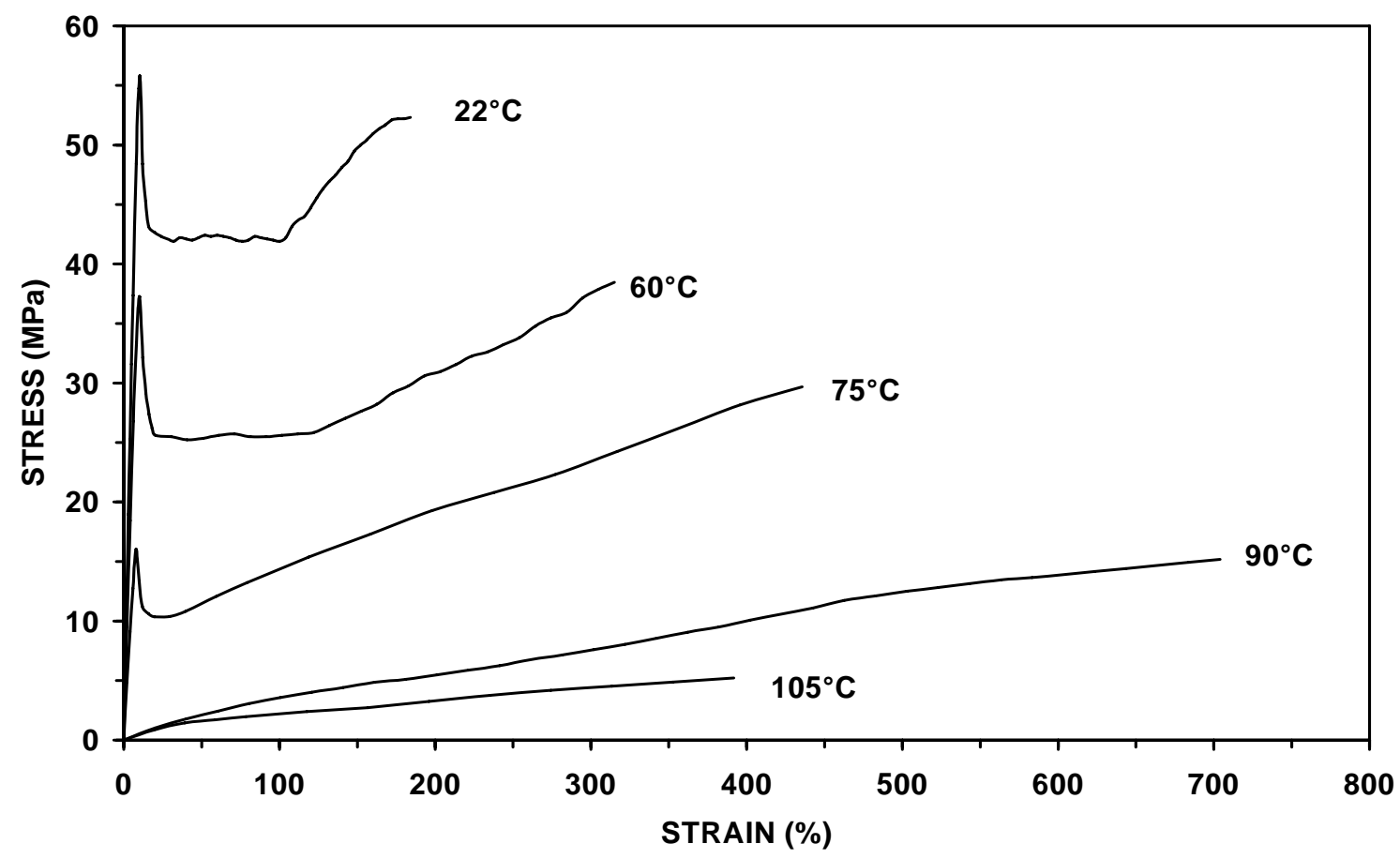

Figure 3: Stress - strain curves for PVC-U

A number of other important points can be taken from Figure 3. It shows how PVC deforms in a rubberlike manner at temperatures above $T_{\mathrm{g}}$. Also, it neatly illustrates how elongation at break (strain) passes through a maximum at around $90^{\circ} \mathrm{C}$. The reduction in elongation at $105^{\circ} \mathrm{C}$ is related to the network structure of PVC. At $100^{\circ} \mathrm{C}$ the least prefect crystallites in the network are melted and so the ability of the network to sustain loading is diminished and elongation is reduced. This effect continues to reduce the elongation at break of PVC up to temperatures $140^{\circ} \mathrm{C}$ to $150^{\circ} \mathrm{C}$, after which elongation at break rises again as sufficient destruction of the PVC network has occurred to allow viscous flow [4].

In the orientation of PVC to enhance properties, whether by uniaxial or biaxial stretching just above $T_{\mathrm{g}}$, the drawing process is terminated before the elongation at break is reached. This has the effect of introducing a residual state of stress into the polymer network. In its simplest form it is assumed that two mechanisms operate to determine behaviour: elastic stretching of the long chains and a viscoelastic resistance to the relative motion of the chains. When the material is stretched above $T_{\mathrm{g}}$ the elastic chain stresses dominate (as the viscoelastic stresses have relaxed), such that if the applied force is removed, the material will recover to its original dimensions. However, if the material is cooled under restraint and the applied force is then removed below $T_{\mathrm{g}}$, the viscoelastic resistance dominates and acts to oppose recovery so that the sheet remains in the stretched state. This means that to produce yielding of the oriented product (at ambient 
temperature) it is now necessary to apply a stress that exceeds the yield stress of the unoriented material (Figure 4). If sufficiently high strain is imposed on rigid PVC by orientation above $T_{\mathrm{g}}$, no necking is observed on subsequent testing of tensile samples at ambient temperature, and the yield point is seen as little more than an inflection point in the load extension curve.

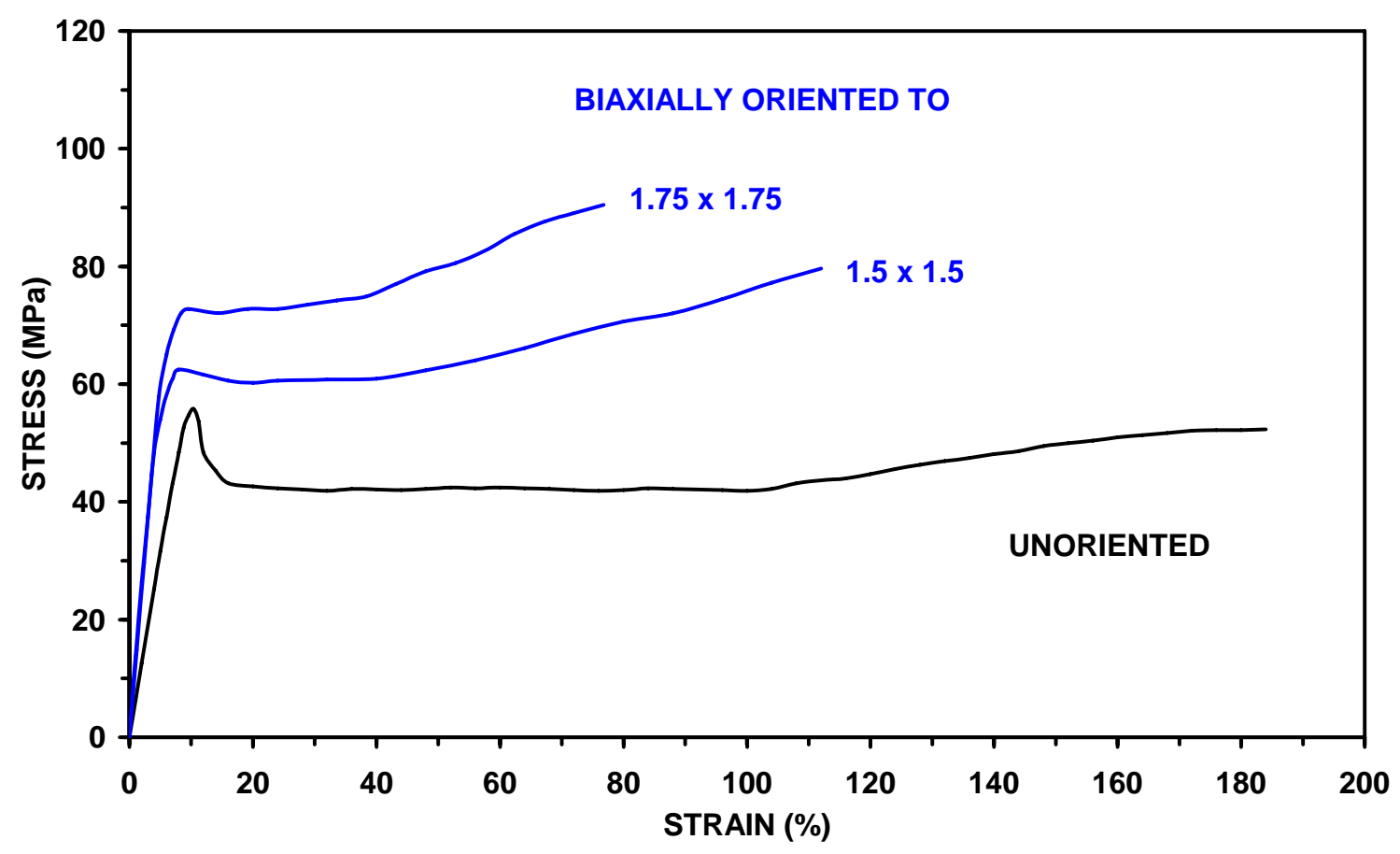

Figure 4: Uniaxial stress strain curves at ambient for rigid PVC sheets biaxially oriented at $90^{\circ} \mathrm{C}$

\section{FILAMENT THEORY}

The content of this section has been drawn from the published work of Miroshnychenko et al. $[10,11]$. Much of the previous work on the mathematical modelling of the uniaxial and/or biaxial deformation of polymers concerns itself with modelling of the deformation process at a temperature above $T_{\mathrm{g}}$. While this remains an essential element for any model to predict stressstrain behaviour of a polymer, the work reported here has attempted to take the modelling a stage further. That is, to develop a model whereby the mechanical behaviour of rigid PVC at room temperature can be predicted after the polymer has been stretched to a prescribed planar deformation above $T_{\mathrm{g}}$.

To achieve the objective the basis of the model has to be capable of determining the residual stress and ideally go on to replicate the various yielding processes undergone by the polymer. For the determination of the residual stress several models reported in the literature were considered [10]. From these, two particular treatments, by Turner [9] and by Arruda and Boyce $[13,14]$, were selected for more rigorous evaluation using experimental data on the deformation of rubberlike materials of Treloar [15] and of Kawabata et al. [16]. The composite model, based on the filament theory of Turner, gave the best correlation, and the filament theory has been used as the foundation of the model to predict the yield stress of oriented PVC.

\subsection{Single filament formulation}


This was proposed by Turner [9] to describe the orientation in polymers. He argued that a single filament could be used to represent the average spatial deformation undergone by the chains within a polymer network. Initially, before stretching, the filament is assumed to be the longest diagonal within a cube (Figure 5(a)) and after drawing filament orientation (Figure 5(b)) can be represented by a unit vector

$$
\mathbf{n}_{\mathrm{f}}=\cos \alpha_{1} \mathbf{n}_{1}+\cos \alpha_{2} \mathbf{n}_{2}+\cos \alpha_{3} \mathbf{n}_{3}
$$

that characterises the direction of the filament with respect to $\mathbf{n}_{1}, \mathbf{n}_{2}$ and $\mathbf{n}_{3}$, which define the stretched frame.

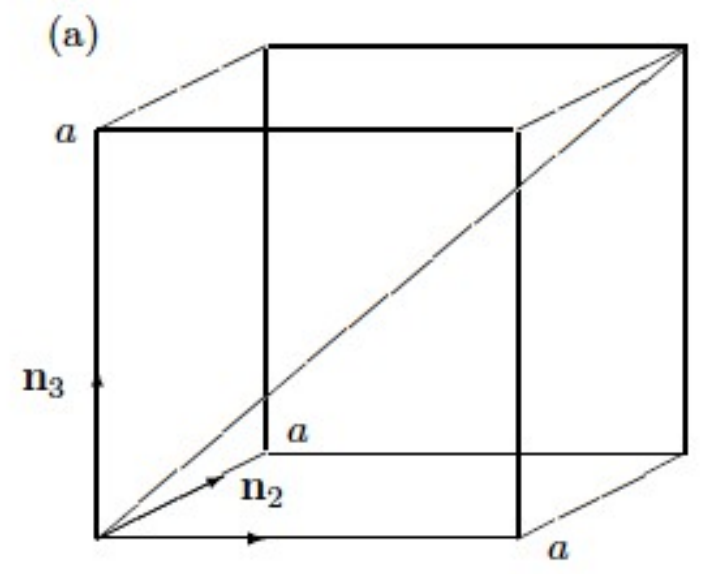

$\mathbf{n}_{1}$

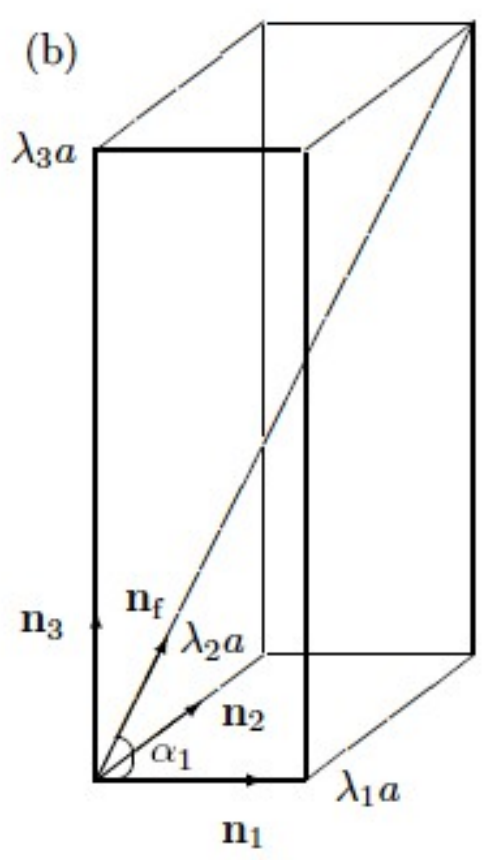

\section{Figure 5: A single filament in (a) unstretched and (b) drawn configurations}

In this configuration, the angle, $\alpha_{i}$, between the filament and a direction of stretch is given by

$$
\cos \alpha_{i}=\frac{\lambda_{i}}{\sqrt{\lambda_{1}^{2}+\lambda_{2}^{2}+\lambda_{3}^{2}}}(i=1,2,3)
$$

and the filament extension, $e_{\mathrm{f}}$, is defined as follows

$$
e_{\mathrm{f}}=\sqrt{\frac{\lambda_{1}^{2}+\lambda_{2}^{2}+\lambda_{3}^{2}}{3}}-1
$$

The tension in the filament, $t_{\mathrm{f}}$, gives rise to the stresses and based upon experimental data Turner proposed an empirical relation for the filament tension 
$t_{\mathrm{f}}=\frac{E_{\mathrm{f}} e_{\mathrm{f}}}{1-e_{\mathrm{f}} / e_{\max }}+t_{\mathrm{p}}$

in terms of filament elasticity parameter (or filament modulus), $E_{\mathrm{f}}$, and limit of filament extensibility, $e_{\max }$. It is clear that the first term in (4) accurately expresses what happens in practice: at low stretches the filament tension is directly proportional to the filament extension and then the filament tension rises steeply as the filament extension approaches its maximum. The second term in (4), $t_{\mathrm{p}}$, has been included here ad hoc. It is the pre-existing tension acting upon the filament that may arise due to factors other than the applied deformation (e.g., processing), which will contribute towards the prediction of the yield stress in oriented PVC.

Principal tensile forces $t_{\mathrm{f} i}$ can be determined directly from their bulk resolution in the deformed configuration (Figure 5 (b))

$t_{\mathrm{f} 1} \mathbf{n}_{1}+t_{\mathrm{f} 2} \mathbf{n}_{2}+t_{\mathrm{f} 3} \mathbf{n}_{3}=t_{\mathrm{f}} \mathbf{n}_{\mathrm{f}}$,

substituting the expression (4) for the filament tension. Thus, true tresses for the filament theory can be expressed in the form

$$
\begin{aligned}
& \sigma_{\mathrm{f} 1}=\frac{t_{\mathrm{f} 1}}{\lambda_{2} \lambda_{3}}=\lambda_{1} t_{\mathrm{f}} \cos \alpha_{1}, \\
& \sigma_{\mathrm{f} 2}=\frac{t_{\mathrm{f} 2}}{\lambda_{1} \lambda_{3}}=\lambda_{2} t_{\mathrm{f}} \cos \alpha_{2}, \\
& \sigma_{\mathrm{f} 3}=\frac{t_{\mathrm{f} 3}}{\lambda_{1} \lambda_{2}}=\lambda_{3} t_{\mathrm{f}} \cos \alpha_{3} .
\end{aligned}
$$

\subsection{Application of the filament theory to predict yield stress in oriented PVC}

To predict the yield stress of an oriented material the anisotropy resulting from the stretching above $T_{\mathrm{g}}$ has to be taken into account (equal biaxial drawing is a specific case of this treatment). During stretching above $T_{\mathrm{g}}$ tension is applied to a filament, which is frozen in the material as a residual stress on cooling. It is assumed that stresses exceeding the residual stress are now required to produce yielding in the oriented material.

If the initial yield stress of an unoriented material is equal to $Y$, then after orientation the principal yield stresses are

$Y_{1}=Y+\sigma_{1}^{\prime}$,

$Y_{2}=Y+\sigma_{2}^{\prime}$,

$Y_{3}=Y+\sigma_{3}^{\prime}$,

where $\boldsymbol{\sigma}^{\prime}$ stand for the "frozen in" stresses. It is the deviatoric part of overall stress $\boldsymbol{\sigma}$ and it can be written as 
$\sigma_{1}^{\prime}=\sigma_{1}-1 / 3\left(\sigma_{1}+\sigma_{2}+\sigma_{3}\right)$

$\sigma_{2}^{\prime}=\sigma_{2}-1 / 3\left(\sigma_{1}+\sigma_{2}+\sigma_{3}\right)$,

$\sigma_{3}^{\prime}=\sigma_{3}-1 / 3\left(\sigma_{1}+\sigma_{2}+\sigma_{3}\right)$.

At this stage, the constitutive equation can be taken in the form

$\boldsymbol{\sigma}=-p \mathbf{I}+\boldsymbol{\sigma}_{\mathrm{f}}$,

in which $p$ is a hydrostatic pressure, $\mathbf{I}$ is the identity tensor, and $\boldsymbol{\sigma}_{\mathrm{f}}$ is the filament stress given by (6).

Hence, these deviatoric stresses (8) can be calculated using (9) and the filament theory (1)-(6) and then used to produce values for $Y_{1}, Y_{2}$ and $Y_{3}$ through (7). Furthermore, the principal yield stresses (7) can be combined to give the yield stress in a particular direction $\theta$ as

$Y_{\theta}=Y_{1} \cos ^{2} \theta+Y_{2} \sin ^{2} \theta$

Yield values in more familiar directions can be determined as $\theta=90^{\circ}$ and $\theta=0^{\circ}$ correspond to the MD and the TD respectively. Thus, if required, the yield strength can be calculated in any direction relative to the main draw directions.

\section{Experimental data on yield stress of oriented PVC}

A series of PVC-U sheets $(120 \mathrm{~mm}$ by $120 \mathrm{~mm}$ squares of $1 \mathrm{~mm}$ thickness) were uniaxially stretched using at $90^{\circ} \mathrm{C}$ and $50 \mathrm{~mm} / \mathrm{min}$ to different draw ratios. After drawing the sheet was cooled to ambient under restraint. Tensile testpieces were then cut from the various sheets and yield properties were measured. The filament theory (1)-(6) depends upon knowledge of a number of physical properties of the material needed to apply the formula (10). In this case, these have been derived [10] through a weighted statistical analysis of the experimental data on yield stress in MD direction shown in Table 1. This resulted in values of $e_{\max }=1.36$, $E_{\mathrm{f}}=2.96 \mathrm{MPa}$ and $t_{\mathrm{p}}=6.97 \mathrm{MPa}$ for the limit of filament extensibility, the filament modulus and the pre-tension respectively. Table 1 also provides a value for the initial yield stress, i.e., $Y=73.7 \mathrm{MPa}$.

Table 1: Yield data for uniaxially oriented PVC-U

\begin{tabular}{|l|c|c|c|c|}
\hline & $\begin{array}{c}\text { Pre-stretch } \\
\text { in MD }\end{array}$ & Number of tests & $\begin{array}{c}\text { Yield stress, } \\
\text { (MPa) }\end{array}$ & $\begin{array}{c}\text { Extension } \\
\text { at yield, (mm) }\end{array}$ \\
\hline \multicolumn{5}{|c|}{} \\
\hline Unstretched & 1.0 & 10 & 73.7 & 2.17 \\
\hline MD & 1.5 & 9 & 72.1 & 1.85 \\
\hline & 2.1 & 8 & 84.3 & 1.91 \\
\hline & 2.5 & 6 & 91.9 & 2.16 \\
\hline \multicolumn{5}{|c|}{} \\
\hline TD & 3.3 & 8 & 108.9 & 1.87 \\
\hline & 1.5 & 11 & 63.4 & 1.85 \\
\hline & 2.2 & 7 & 68.4 & 1.87 \\
\hline & 2.4 & 6 & 68.3 & 1.84 \\
\hline
\end{tabular}


The predicted yield stress values are plotted as continuous lines in Figure 6. The line for properties in the MD of uniaxially oriented sheet lies close to the measured yield properties (solid squares): an expected result as the data was used to generate the material parameters for the filament theory. There is reasonably good correlation between the predicted and measured yield values (unfilled squares) in the TD for uniaxially oriented sheet. It appears from Figure 6 that measured yield values at a planar strain of 1.5 are inconsistent with the other measured data. This may have been due experimental error (unlikely as the results of 9 tests contributed to the calculated average) or more likely the original sheet had relatively poor properties prior to stretching (a factor that has been experienced on a number of occasions during the work on orientation of PVC at IPTME). Discarding the uniaxial data point in the MD for the pre-stretch of 1.5 may well have improved the quality of the predicted yield properties. However, even with its inclusion the really positive result that can be seen from Figure 6 is that the filament theory reproduces the systematic pattern of yield stress as a function of planar strain. The core line of the plot represents the predicted yield stress for equally biaxially oriented PVC and its branches correspond to the predicted yield stress of unequally biaxially oriented sheets in both the major and minor draw directions. Note: as well as determining properties in MD and TD (Table 1), yield stress was also measured in the directions of $\theta=60^{\circ}$ and $\theta=30^{\circ}$ over a similar range of uniaxial draw ratios [10]. Yield stress values predicted by equation (10) correlated well with this experimental data.

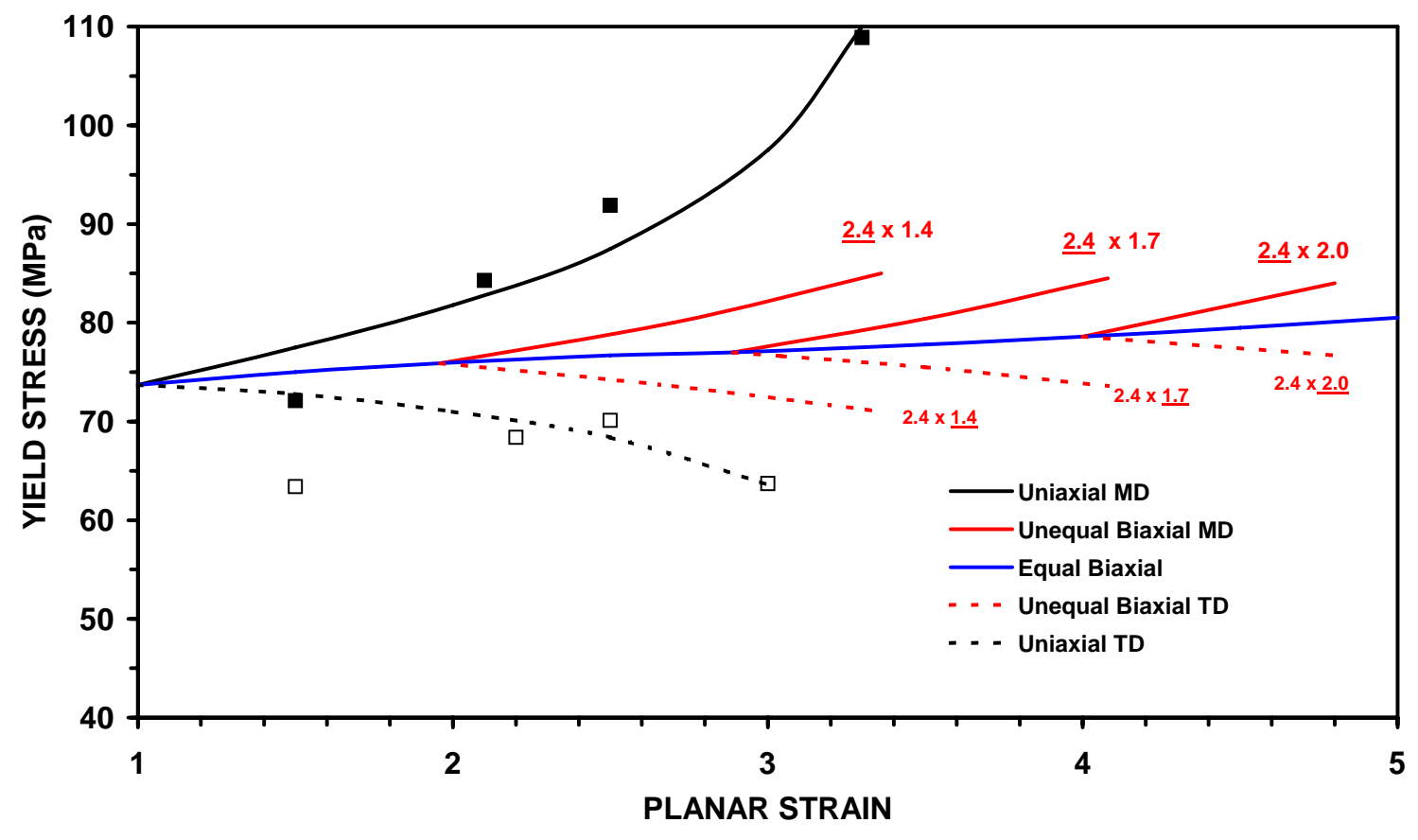

Figure 6: Prediction of yield stress for oriented rigid PVC: $\square$ and $\square$ represent measured yield values of uniaxially oriented sheets in the MD and TD respectively.

\section{COMPOSITE MODEL AND FURTHER DEVELOPMENTS}

In a recent paper, Miroshnychenko et al. [11] have developed the filament theory further by coupling it to the approach of Poisson's function [17] to form thus the "composite" model. In the development of the composite model a different expression for filament tension (4) has been proposed [11] and in its final refined form it can be written 
$t_{\mathrm{f}}=\frac{E_{\mathrm{f}} \lambda_{\mathrm{f}}}{1-\lambda_{\mathrm{f}} / \lambda_{\max }}$

where $\lambda_{\mathrm{f}}$ is the filament stretch (with $\lambda_{\max }$ being its maximum value) given by

$\lambda_{\mathrm{f}}=\sqrt{\frac{\lambda_{1}^{2}+\lambda_{2}^{2}+\lambda_{3}^{2}}{3}}$.

This expression (11) conveys the asymptotic behaviour of polymer material in the large-tension limit more neatly than (4).

The concept of the composite model is to have a linear element, $\boldsymbol{\sigma}_{\mathrm{p}}$, that accurately describes the deformation of a polymer network at low strains (the approach of Poisson's function) coupled with a non-linear element, $\boldsymbol{\sigma}_{\mathrm{f}}$, that begins to exert an influence at moderate stretches and then dominates at large deformations (the filament theory). Thus, the overall stress, $\boldsymbol{\sigma}$, can be expressed in the additive form

$\boldsymbol{\sigma}=-p \mathbf{I}+\boldsymbol{\sigma}_{\mathrm{p}}+\boldsymbol{\sigma}_{\mathrm{f}}$

The composite model [11] allows us to predict the stress-strain relations for elastomeric materials more accurately than either the filament theory (9) alone or the eight-chain model [13].

\subsection{Approach of Poisson's function}

Turner and Brennan [17] considered the stresses in the area of low strain: starting from the wellknown stress-strain relationships of linear elasticity

$\sigma_{1}-v\left(\sigma_{2}+\sigma_{3}\right)=E\left(\lambda_{1}-1\right)$,
$\sigma_{2}-v\left(\sigma_{3}+\sigma_{1}\right)=E\left(\lambda_{2}-1\right)$,
$\sigma_{3}-v\left(\sigma_{1}+\sigma_{2}\right)=E\left(\lambda_{3}-1\right)$,

where $\sigma_{i}$ and $\lambda_{i}$ are the principal true stresses and principal stretches respectively, and $E$ is Young's modulus. Poisson's ratio is one of the core concepts of linear elasticity, and for incompressible materials $\lambda_{1} \lambda_{2} \lambda_{3}=1$, it is constant, i.e., $v=1 / 2$. In order to extend the applicability of (14) into finite elasticity, Turner and Brennan invoked the concept of a Poisson's function, which corresponds to a variable Poisson's ratio. This concept is not new and its relation to Poisson's ratio has been reported [18].

For thin polymer sheets, one stress (say, $\sigma_{3}$ ) can be considered as negligible and taken to be zero. After some manipulation of the equations the stress-strain relations can be written in terms of the difference of two stresses

$$
\begin{gathered}
\sigma_{\mathrm{p} 1}-\sigma_{\mathrm{p} 3}=E \frac{\lambda_{1}-\lambda_{3}}{1+v}, \\
\sigma_{\mathrm{p} 2}-\sigma_{\mathrm{p} 3}=E \frac{\lambda_{2}-\lambda_{3}}{1+v},
\end{gathered}
$$


in which the Poisson's function, $v$, is given by

$$
v=\frac{1-\lambda_{3}}{\lambda_{1}+\lambda_{2}-1-\lambda_{3}} .
$$

However, due to setting $\sigma_{3}$ to zero, the Poisson's function (16) is asymmetrical (i.e., it is disadvantaged in the $\lambda_{3}$ stretch direction). Thus, Miroshnychenko et al. [11] proposed that $v$ could be approximated by the interpolation formula

$$
v \approx \frac{1}{\lambda_{1}+\lambda_{2}+\lambda_{3}-1}
$$

which is symmetrical in all three principal stretches and follows closely the limiting behaviour of (16).

\subsection{Constitutive equations for the composite model}

The stresses arising from the approach of Poisson's function (15) and from the filament theory (6) constitute the composite model (13) and can be stated in terms of the difference of two stresses

$$
\begin{aligned}
& \sigma_{1}-\sigma_{3}=E \frac{\lambda_{1}-\lambda_{3}}{1+v}+\frac{E_{\mathrm{f}} \lambda_{\mathrm{f}}}{1-\lambda_{\mathrm{f}} / \lambda_{\max }} \cdot \frac{\lambda_{1}^{2}-\lambda_{3}^{2}}{\sqrt{\lambda_{1}^{2}+\lambda_{2}^{2}+\lambda_{3}^{2}}}, \\
& \sigma_{2}-\sigma_{3}=E \frac{\lambda_{2}-\lambda_{3}}{1+v}+\frac{E_{\mathrm{f}} \lambda_{\mathrm{f}}}{1-\lambda_{\mathrm{f}} / \lambda_{\max }} \cdot \frac{\lambda_{2}^{2}-\lambda_{3}^{2}}{\sqrt{\lambda_{1}^{2}+\lambda_{2}^{2}+\lambda_{3}^{2}}} .
\end{aligned}
$$

The model now depends upon knowledge of three material parameters: the elastic modulus, $E$, the filament modulus, $E_{\mathrm{f}}$, and the maximum filament stretch, $\lambda_{\max }$, whilst $v$ is not a material constant but a variable (17) depending upon the three principal stretches. The model exhibits non-linear elastic behaviour at high strains: an intrinsic property of elastomeric material. The composite model has been able to accurately reproduce the experimental data of Treloar [15] and Kawabata et al. [16]. However, to date it has yet to be tested with data on the properties of oriented PVC.

Further improvement to the composite model (18) can be achieved by proposing a relationship between the elastic modulus, $E$, of the linear element, and two other parameters, $E_{\mathrm{f}}$ and $\lambda_{\max }$, of the non-linear part. Such a relationship would reflect common macromolecular origin (nature) of linear elastic deformation and of orientation and limiting extensibility in polymer chains. One such relation was proposed in [11],

$$
E=E_{\mathrm{f}} \lambda_{\max }
$$

and the composite model (18) complemented by (19) has been named "the filament model" and it has shown considerable promise.

\section{DISCUSSION AND CONCLUSIONS}


It can be seen that the equations of the filament theory are quite simple mathematically and the theory has complied with the prime objective of the work: it can be used to predict the systematic variation of yield stress of oriented rigid PVC as a function of imposed planar strain. The use of the theory does depend upon knowing certain properties of the material. However, these can be derived from the yield properties of the unoriented material and from a small number of uniaxially oriented sheets. This provides the prospect of being able to generate a complete understanding of how any combination of draw ratios might affect the yield strength of any oriented PVC formulation from the results of a limited number of experiments. This could be extremely valuable when selecting draw ratios for biaxially oriented products, such as pipes, as combinations that produce poor mechanical strength in the minor draw direction can be identified and avoided.

To have complete confidence in the filament theory, its predictive capability needs to be tested with further sets of yield data for a range of oriented rigid PVC formulations. At the same time it would be extremely interesting to evaluate the refined composite model (18) and to assess whether the composite and filament models improve the accuracy of predictions for the yield stress in oriented PVC. From that point, the next step would be to address the question: can the composite and filament models predict the strength at break values for oriented PVC as a function of imposed planar strain? Intuitively, the answer would be affirmative, as the tendency for strength at break (Figure 2) is similar to the tendency for yield strength (Figure 1).

\section{Acknowledgements:}

The authors would like to thank Professor Marianne Gilbert (IPTME, Loughborough University) and Dr Tony Green (Department of Mathematical Sciences, Loughborough University) for their guidance and valuable contributions during the research at IPTME. Also, the support given to the project by Dr Tony Day (Hydro Polymers Ltd) and by Dr Les Holloway (Wavin Industrial Products Ltd) was greatly appreciated.

\section{References}

1. S.H. Pinner, Plastics, 82-85, May 1965, and 220-226, June 1965.

2. A.J. DeVries and C. Bonnebat, Polymer Engineering and Science, 16, 93-100, 1976.

3. B. Terselius, PhD Thesis, The Royal Institute of Technology, Stockholm, 1983.

4. D.J. Hitt and M. Gilbert, Materials Science and Technology, 8, 739-745, 1992.

5. D.J. Hitt, Proceedings of the IOM International Conference, PVC 96 "new perspectives", Brighton, 1996, p. 97-108.

6. M. Gilbert, Z. Liu and D.J. Hitt, Polymer Engineering and Science, 37, 1858-1864, 1997.

7. D.J. Hitt and M. Gilbert, Plastics, Rubber and Composites, 29, 149-160, 2000.

8. D.J. Hitt and M. Gilbert, Journal of Applied Polymer Science, 89, 3859-3867, 2003.

9. D.M. Turner, Workshop on Deformation Modelling for Solid Polymers, Oxford University, July 1997, p. 17.

10. D. Miroshnychenko, MPhil Thesis, Loughborough University, UK, 2002.

11. D. Miroshnychenko, W.A Green and D.M. Turner, Journal of the Mechanics and Physics of Solids, 53, 748-770, 2005.

12. T.E. Brady, Polymer Engineering and Science, 16, 638-644, 1976.

13. E.M. Arruda and M.C. Boyce, Journal of the Mechanics and Physics of Solids, 41, 389412, 1993.

14. M.C. Boyce and E.M. Arruda, Rubber Chemistry and Technology, 73, 504-523, 2000.

15. L.R.G. Treloar, Transactions of the Faraday Society, 42, 59-70, 1944. 
16. S. Kawabata, M. Matsuda, K. Tei and H. Kawai, Macromolecules, 14, 154-162, 1981.

17. D.M. Turner and M Brennan, Plastics and Rubber Processing and Applications, 14, 183188, 1990.

18. M.F. Beatty and D.O. Stalnaker, Journal of Applied Mechanics, 53, 807-813, 1986. 